\title{
Fabrication of organic light-emitting diode using molybdenum trioxide interlayer between electrode and organic interface
}

\author{
DHRUBAJYOTI SAIKIA* and RANJIT SARMA \\ Thin Film Laboratory, Department of Physics, J.B. College, Jorhat 785001, India \\ *Author for correspondence (dhrubajun@gmail.com)
}

MS received 6 April 2017; accepted 27 June 2017; published online 11 July 2018

\begin{abstract}
In this study, high-performance of organic light-emitting diodes (OLEDs) with a buffer layer of $\mathrm{MoO}_{3}$ is demonstrated. With an optimal thickness of $\mathrm{MoO}_{3}(12 \mathrm{~nm})$, the luminance efficiency is found to be increased compared to the single layer anode OLED. To study the influence of $\mathrm{MoO}_{3}$ buffer layer on OLED performance, we deposited $\mathrm{MoO}_{3}$ films with different thicknesses on the fluorine-doped tin oxide (FTO) surface and studied J-V and L-V characteristics of the OLED devices. Also, further analysis was carried out by measuring sheet resistance, optical transmittance and surface morphology with the FESEM images. Here, we found that $\mathrm{MoO}_{3}(12 \mathrm{~nm})$ buffer layer is a good choice to increase the efficiency of FTO-based OLED devices within the tunnelling region. Here, the maximum value of current efficiency is $6.15 \mathrm{~cd} \mathrm{~A}^{-1}$.
\end{abstract}

Keywords. Hole-injection layer; OLED; surface resistance; optical transmittance; FESEM.

\section{Introduction}

Organic light-emitting diodes (OLEDs) have drawn enormous interest with the flat panel display because it has many advantages, such as a simple fabrication process, a quick switching speed, a wide-viewing angle and low-fabrication cost compared to the conventional LCD. The rapid development of organic electronics is a result of a large-scale, rigorous effort from the science and engineering research community. Interfacial engineering was one of the major areas of development of OLED. Therefore, great efforts were made to improve their performance by modifying the interface structure to achieve an effective and balanced injection of the charge carriers [1,2]. As a result, OLEDs are currently the most advanced devices in the field of organic electronics. Therefore, to enhance the device efficiency of OLEDs, it is necessary to develop more optimized device architecture. Especially, improving the injection of charge carriers from the electrode to light emitting layer is necessary to achieve highly efficient OLEDs. The interface between the organic layer and the electrodes plays a significant role in the OLED performance, because it determines the efficiency of the charge carrier injection from the electrode into the light emitting layer [3]. The insertion of a buffer/injection layer between anode and hole-transport layer (HTL) is one of the simple and efficient methods to improve device performance. $\mathrm{Mu}$ et al [4] reported an OLED using both copper (II) phthalocyanine and PEDOT as a hole buffer layer with maximum efficiency of $8.2 \mathrm{~cd} \mathrm{~A}^{-1}$. Meng and his co-workers [5] fabricated OLEDs on bilayer graphene electrode. Daeil Kim [6] studied the effect of the $\mathrm{TiO}_{2}$ buffer layer on the properties of ITO films. Also, Chien-Jung Huang et al [7] worked on flexible OLED by the insertion of ultrathin $\mathrm{SiO}_{2}$ buffer layers, in which they obtained efficiency $>8 \mathrm{~cd} \mathrm{~A}^{-1}$. Thus, several approaches were employed to overcome relatively high hole-injection barrier between the electrode and the organic hole-transport layers, which include different materials such as transition metal oxide [8,9], small organic molecule [10] and conducting polymers $[11,12]$. The presence of such injection layer enhanced the hole injection into the organic layers and also improves the surface roughness of the electrode, which directly affects the device performance including its turn on voltage. Inorganic insulating buffer layers, such as $\mathrm{LiF}$ [13], $\mathrm{Al}_{2} \mathrm{O}_{3}$ [14], $\mathrm{SiO}_{2}$ [7] and $\mathrm{V}_{2} \mathrm{O}_{5}[15,16]$, were reported in the literature. Zhang and his co-workers [17] calculated the $\mathrm{J}-\mathrm{V}$ characteristics by using sodium stearate $(\mathrm{NaSt})$ buffer layer based on the WKB (Wentzel-Kramers-Brillouin) approximation method. Lu and Yokoyama [18] reported hole block effect due to the insertion of $\mathrm{Ta}_{2} \mathrm{O}_{5}$ and $\mathrm{HfO}_{2}$ buffer layers, whereas other reports also showed enhancement of device performance with the introduction of various buffer layers, such as $\mathrm{ZnPc}$ [19], tungsten oxide [20,21], Adenine [22], etc. Akanksha Uniyal and Poornima Mittal [23] provide a comparison of the bilayer and multilayer OLED. Yu-Long Wang et al [24] used tetramethyl substituted copper (II) phthalocyanine as a hole-injection enhancer in OLED. More recently, Xu et al [25] developed a multilayer transparent conducting electrodes for OLEDs, while Meiling Shan et al [26] reported an enhanced hole injection in OLEDs utilizing a copper iodidedoped hole-injection layer. Similarly, molybdenum trioxide $\left(\mathrm{MoO}_{3}\right)$ was also used as a modification of anode surface to enhanced hole injection [27] by using multiple quantum well 
structure in a hole-transport layer. However in the literature, all the reports were found to be based on ITO (Indium tin oxide) electrode. On the other hand, fluorine-doped tin oxide (FTO) is cost effective than the ITO, it is not widely used to fabricate OLEDs because of its less transparency and conductivity than ITO [28]. However, there is a published report that indium has a tendency to diffuse into the light-emissive layer under device operation [29], which may in turn influence the quantum efficiency and lifetime of OLEDs. On the other hand, in the case of FTO anode, there is no indium present for possibilities of diffuse into the emissive layer in the OLED. Also, the chemical composition as well as the work function of FTO was found to be independent of the cleaning methods employed [30], which is opposite to ITO. It indicates that FTO is more stable to oxidation than ITO. Therefore, some material of intermediate layers such as PEDOT: PSS (poly(3,4-ethylenedioxythiphene) : poly(styrenesulfonate)) and sulphonated polyaniline can be used to reduce the potential barrier for FTO-based OLED [31,32]. But till now, no report was found to be published on the effect of molybdenum trioxide $\left(\mathrm{MoO}_{3}\right)$ over non-conventional FTO surface. $\mathrm{MoO}_{3}$ is a transition metal oxide which forms a better ohmic contact with TPD layer than that of single FTO surface. It can be evaporated easily at relatively mild temperature during vacuum evaporation of device fabrication. Also, $\mathrm{MoO}_{3}$ has several advantages such as less contamination, energy level matching with organic molecules and significantly reduces the operational voltage and improves the device efficiency $[33,34]$. Hence, in this study, we address an OLED, which is fabricated on FTO-coated glass by thermal vacuum deposition method and optimize it by using bilayer anode at various thicknesses of the $\mathrm{MoO}_{3}$ film, which are characterized in terms of their efficiency calculation.

\section{Experimental}

In this study, all devices were fabricated on FTO-coated glass and thermally deposited AL (aluminium) was used as the cathode. The FTO glasses were ultrasonically cleaned with acetone, isopropanol and deionized water for $15 \mathrm{~min}$ and then dried by an air gun before fabrication of OLED. This cleaning step is used to remove the surface contaminants and to provide a clean FTO surface to enhance the adhesion of another layer onto its substrates. In this study, the filaments and boats are also flash-cleaned in vacuum by passing a heavy current momentarily. Flash-cleaning of filaments and boats are essential for removal of contaminants adhering to them. On the other hand, before OLED fabrication, FTO sheets were prepatterned using etching process (combination of zinc dust and dilute $\mathrm{HCl}$ ). Then, all the FTO sheets are annealed at $572 \mathrm{~K}$ to improve the optical performance. The thermal annealing process enhanced the optical properties of transparent conducting oxide by enhancing the surface properties and the bandgap of intraband electronic transitions $[35,36]$. Here, all the depositions are carried out at a pressure $<2 \times 10^{-6}$ Torr.
All the organic and inorganic layers were evaporated at the deposition rate $>10 \AA \mathrm{s}^{-1}$. All the devices were fabricated by using thermal vacuum evaporation unit (TVEU) and the corresponding film thickness was recorded by a digitalized and interconnected thickness monitor (Model DTM-10). Finally, we store the sample in a vacuum oven for $20 \mathrm{~min}$ to stabilize before characterization. Sheet resistance and optical transmittance are measured by four probes resistivity method and UV-visible double beam spectrophotometer unit. The current-voltage-luminance characteristics of the fabricated OLEDs were measured by digitally controlled source-meter (SMU) and luminance meter unit. Electroluminescence (EL) spectra of the devices are recorded by a PR650 spectrometer unit. All tests are performed in air at room temperature under dark room conditions without any encapsulation and all materials are purchased from Sigma-Aldrich (USA) and used without further purification. Figure 1a represents the schematic representation, figure $1 \mathrm{~b}$ represents the energy level alignment and figure $1 \mathrm{c}$ provides a photograph of OLED device.

\section{Result and discussion}

We have fabricated the OLED samples using $\mathrm{MoO}_{3}$ as a hole-injection layer (HIL), $N, N^{\prime}$-bis(3-methyle phenyl)$N, N^{\prime}$ (phenyl)-benzidine (TPD), Tris (8-hydroxy quinolinato) aluminium $\left(\mathrm{Alq}_{3}\right)$ and lithium fluoride $(\mathrm{LiF})$ were used as a hole-transport layer (HTL), emitting layer and an electron transport layer (ETL), respectively, and compared their J-V-L characteristics of respected OLEDs with different thicknesses of HIL and also that of HIL-free sample. The output characteristics of all the OLED devices with buffer thicknesses of $4,8,12$ and $16 \mathrm{~nm}$ are shown in figure 2. The structures of the bottom light-emitting OLEDs used in this study are given below:

Device 1: FTO/ $\mathrm{MoO}_{3}(0 \mathrm{~nm}) / \mathrm{TPD}(35 \mathrm{~nm}) / \mathrm{Alq}_{3}(45 \mathrm{~nm}) /$ Lif $(5 \mathrm{~nm}) / \mathrm{Al}(100 \mathrm{~nm})$,

Device 2: $\mathrm{FTO} / \mathrm{MoO}_{3}(4 \mathrm{~nm}) / \mathrm{TPD}(35 \mathrm{~nm}) / \mathrm{Alq}_{3}(45 \mathrm{~nm}) /$ Lif $(5 \mathrm{~nm}) / \mathrm{Al}(100 \mathrm{~nm})$,

Device 3: $\mathrm{FTO} / \mathrm{MoO}_{3}(8 \mathrm{~nm}) / \mathrm{TPD}(35 \mathrm{~nm}) / \mathrm{Alq}_{3}(45 \mathrm{~nm}) /$ Lif $(5 \mathrm{~nm}) / \mathrm{Al}(100 \mathrm{~nm})$,

Device 4: FTO/ $\mathrm{MoO}_{3}(12 \mathrm{~nm}) / \mathrm{TPD}(35 \mathrm{~nm}) / \mathrm{Alq}_{3}(45 \mathrm{~nm}) /$ Lif $(5 \mathrm{~nm}) / \mathrm{Al}(100 \mathrm{~nm})$,

Device 5: FTO/ $\mathrm{MoO}_{3}(16 \mathrm{~nm}) / \mathrm{TPD}(35 \mathrm{~nm}) / \mathrm{Alq}_{3}(45 \mathrm{~nm}) /$ Lif (5 nm)/Al (100 nm).

Figure 3 represents the transmittance spectra of bilayer anode for the $\mathrm{MoO}_{3}$ layer over FTO electrode. Here, the maximum value of optimized $\mathrm{FTO} / \mathrm{MoO}_{3}$ bilayer anode is $>80 \%$ (average value of $83.85 \%$ within $530-560 \mathrm{~nm}$ wavelength region), which is a good indicator for transparent electronics. Similarly, the variation of sheet resistance $v s$. thickness of 


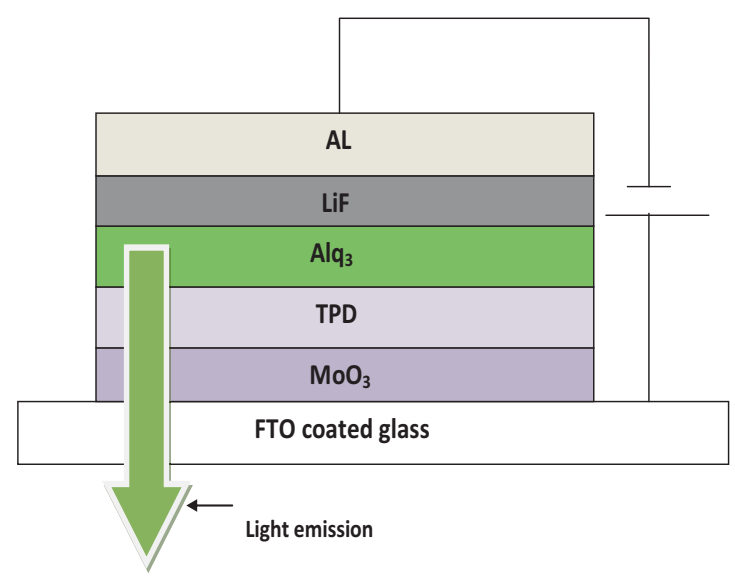

(a)

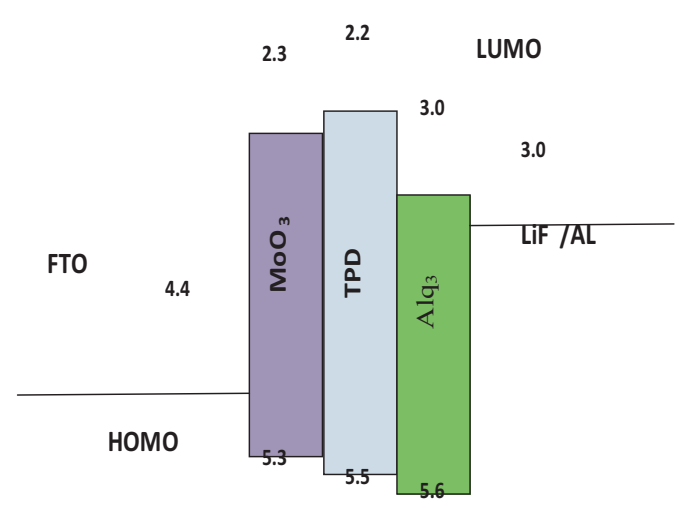

(b)

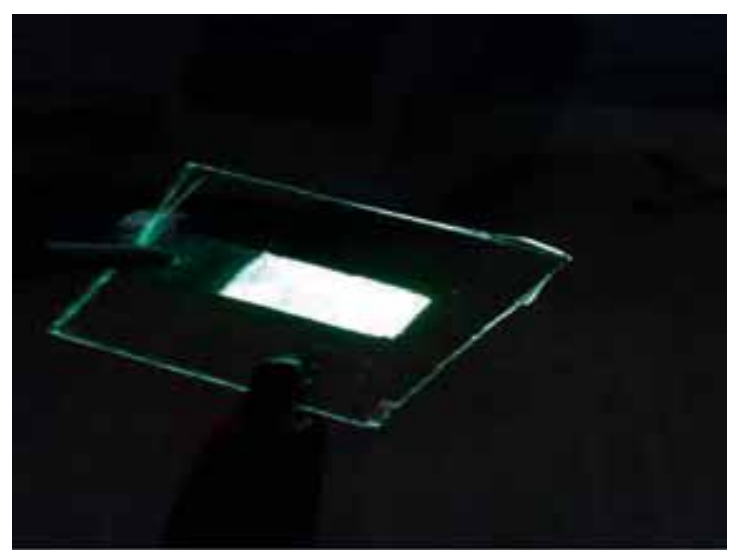

(c)

Figure 1. (a) Schematic representation of OLED structure, (b) energy band structure of OLED device and (c) photograph of OLED.

$\mathrm{MoO}_{3}$ layer over FTO surface is also shown here. This shows a decreasing trend of surface resistance at a higher thickness of buffer layer [37].

The current-voltage and the luminance-voltage characteristics of OLEDs with configuration FTO/buffer layer/TPD $(35 \mathrm{~nm}) / \mathrm{Alq}_{3}(45 \mathrm{~nm}) / \mathrm{Lif}(5 \mathrm{~nm}) / \mathrm{Al}(100 \mathrm{~nm})$ is shown in figure $2 \mathrm{a}$ and $\mathrm{b}$. In this study, we varied the operational voltage from 0 to $21 \mathrm{~V}$. It was found that OLEDs with a $\mathrm{MoO}_{3}$ layer at $12 \mathrm{~nm}$ thickness have a relatively higher performance at the same applied voltage compared with other devices.

To study the influence of thickness variation of molybdenum trioxide $\left(\mathrm{MoO}_{3}\right)$ on luminance, we kept the thickness of all layers constant except this layer, which was varied between 4 and $16 \mathrm{~nm}$. It is found that when the thickness of the buffer is continuously increased, then, there is a decreasing tendency of current density. This indicated that this interlayer has the direct effect of blocking the hole current by controlling the flow of positive charge carrier. On the other hand, a good
EL device should possess high luminance efficiency. In this study, it is found that the device with $12 \mathrm{~nm} \mathrm{MoO}_{3}$ buffer layer has the highest efficiency of $6.15 \mathrm{~cd} \mathrm{~A}^{-1}$ compared to the other OLED devices. Therefore, current efficiency increases compared to the device without a buffer layer $\left(3.80 \mathrm{~cd} \mathrm{~A}^{-1}\right)$. With the increasing thickness of buffer layer, there is a gradual decrease of luminance and increase in the efficiency is observed, which is due to the blocking of positive charge carrier current by the high thickness of buffer layer [38]. This improvement in efficiency is because of the proper balancing of charge carrier injection. Therefore, it can be said that insertion of interlayer enhanced the efficiency by blocking the hole injection. This interlayer also prevents the diffusion of metal and oxygen into the organic layer from the anode and hence, reduces the probability of an electrical breakdown of the device. From the literature, it is known to us that the mobility of electron is lower in electron transport layer than the mobility of holes in the hole-transport layer [39]. As a result of which, there is an accumulation of positive charge carrier 


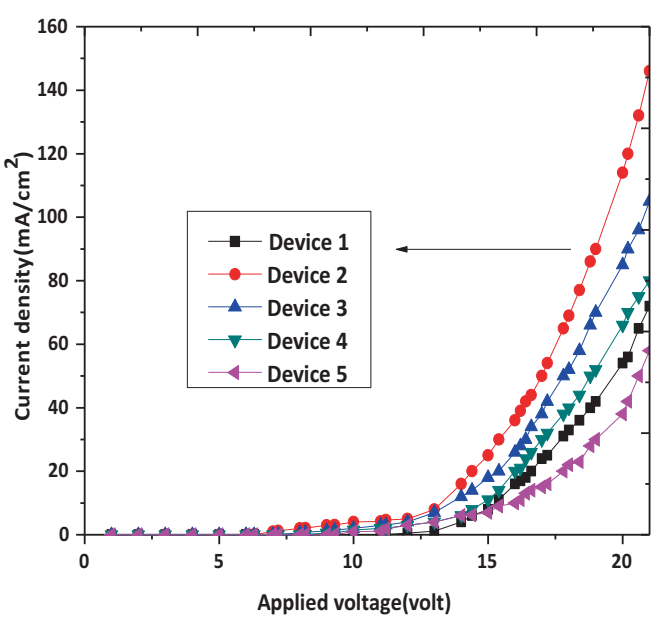

(a)

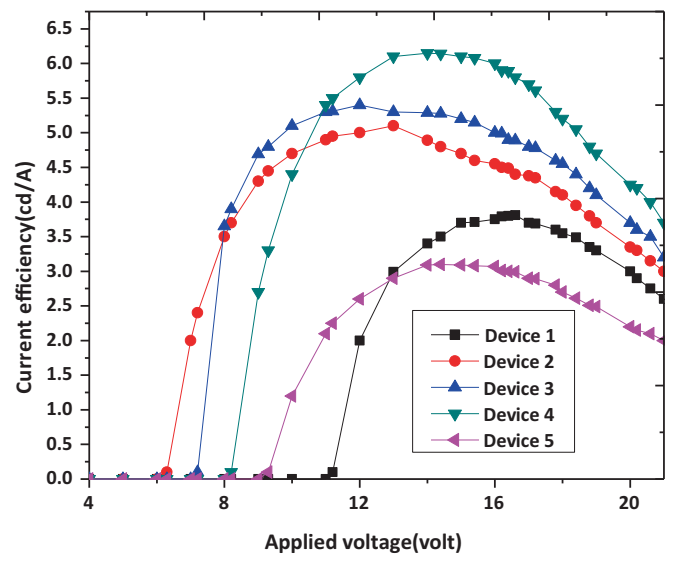

(c)

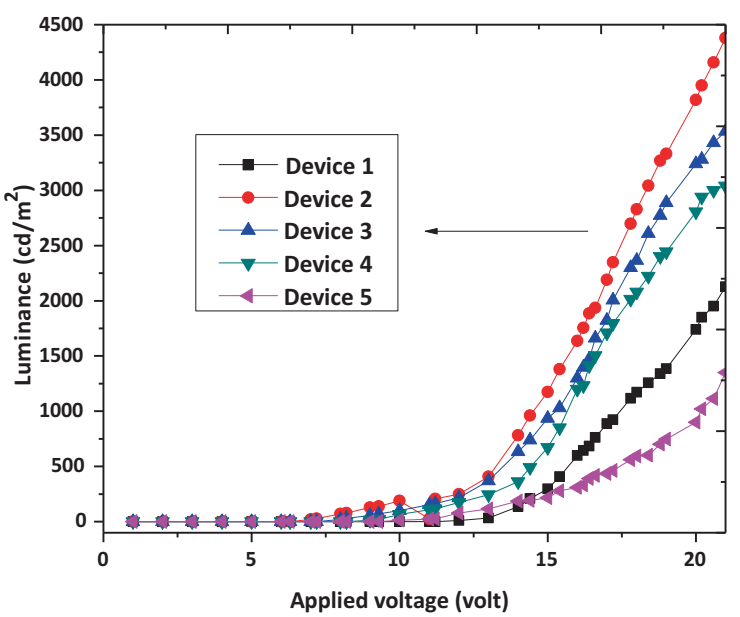

(b)

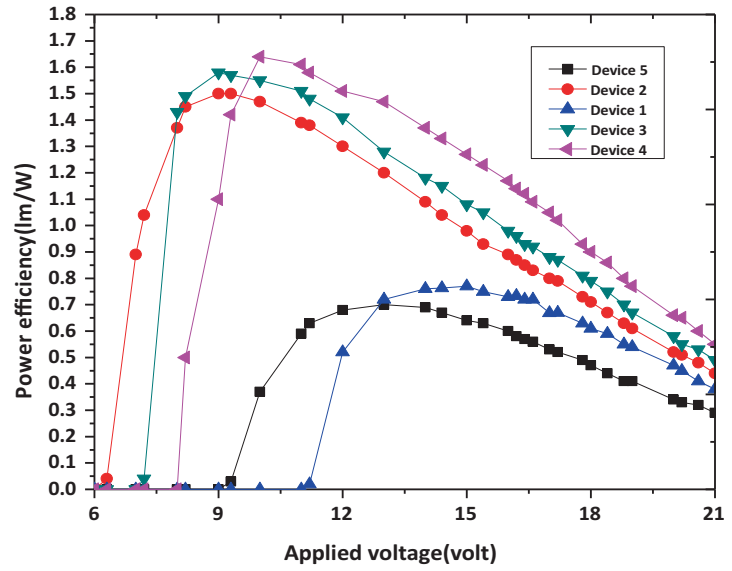

(d)

Figure 2. Graphs of (a) voltage vs. current density, (b) voltage $v s$. luminance, (c) voltage $v s$. current efficiency and (d) voltage $v s$. power efficiency.

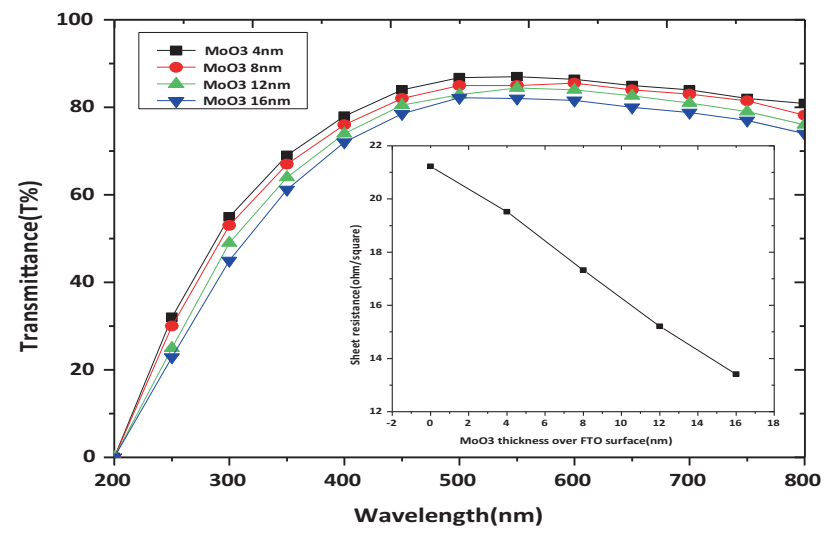

Figure 3. Transmission spectrum and sheet resistance of bilayer FTO/ $\mathrm{MoO}_{3}$ film.

at the interface of HTL/ETL layer. Therefore, to enhance the efficiency of the device, we should either decrease the number of positive charge carrier mobility or increase the electron mobility. In our case, we balance the mobility of positive charge carrier by using the $\mathrm{MoO}_{3}$ buffer layer between the FTO and TPD layers.

In other words, this interlayer can help in preventing the accumulation of excess holes in the luminance layer and thus, increasing the probability of electron-hole pair recombination. Initially, when the thickness of buffer layer is thin, mobility of the positive charge carrier is significantly increased due to the increase in charge tunnelling from the FTO surface to the TPD layer. This effect is associated with the high value of current density and luminance of the device along with the lower threshold voltage. Then, we increase the thickness of the buffer layer continuously, so that there is a decreasing tendency of both current density and luminance of the devices. This may be due to the reducing of tunnelling effect between the anode and hole-transport layer with increase in buffer thickness. From the characteristic graph shown in figure $2 \mathrm{a}-\mathrm{c}$, it is clear that although the values of current density and luminance are decreased, their efficiency is more improved continuously with the increase in the thickness of buffer layer. In this study, maximum efficiency is provided by device 4 with the optimized thickness 
of $12 \mathrm{~nm}$. Then, the efficiency of the device is decreased due to the reduction of tunnelling effect at the higher thickness of this buffer layer. The initial increasing of luminance may be due to the minimized crystal defects within the interface region of FTO and TPD layers, because of the presence of oxide layer. Also, when we placed the $\mathrm{MoO}_{3}$ layer between the anode and hole-transport layer, then, there is a high tendency for the positive charge carrier to inject into the organic layer from a high energy level compared with that of without any buffer layer as shown in figure $1 \mathrm{~b}$. There is also some hole trap in the hole-transport layer which should trap the holes before they reach the light emitting region. When the holes are injected relatively from a higher energy level, then, the probability of their trapping is decreased. This is the reason for increase in the device efficiency with the presence of buffer layer than the device without any buffer layer. Figure $2 \mathrm{c}$ represents the variation of current efficiency of the OLED devices with respect to their applied voltage. In this figure, it is clear that device 4 shows the maximum efficiency and device 5 has the lowest efficiency. This situation is directly linked with the proper charge injection process i.e., exciton formation probability. When the thickness is very low, the charge balancing process is slightly enhanced. But, when we increase the thickness, this process tends to enhance more, because of which we get better device efficiency in our devices. This effect is maximum at the optimized thickness of $12 \mathrm{~nm}$ (device 4), where we get the highest efficiency of $6.15 \mathrm{~cd} \mathrm{~A}^{-1}$. This implies that at a $12 \mathrm{~nm}$ thickness of buffer layer, there is more balance of positive charge injection with regard to negative charge from the anode side of the device. But after the optimized thickness, efficiency decreases. This is due to the reducing of charge carrier tunnelling at a higher thickness of the buffer layer from the electrode to the hole-transport layer. Similarly, the variation of power efficiency $v s$. applied voltage characteristics for all the devices is shown in figure $2 \mathrm{~d}$, in which device 4 shows the highest value where the maximum balance of holes and electron injection takes place.

It is found that the power efficiency $(\eta)$ and current efficiency $(\gamma)$ are depending on each other, and their relation can be given by the following equation of Lambertian emission pattern [40].

$$
\eta=\pi \frac{L}{V J}=\pi \frac{1}{V}\left(\frac{L}{J}\right)=\pi \gamma \frac{1}{V}
$$

where $L$ is the luminance measured from the light emitting surface and $V$ the applied voltage. The value of current efficiency depends on the optical coupling and the internal quantum efficiencies [41]. From this equation, it is clear that $\eta$ is inversely proportional to $V$.

Figure 4 shows the EL spectrum of all the OLED devices in this study is $16 \mathrm{~V}$, which is measured by a spectrometer unit. It can be seen that all the five devices have the maximum peak in their EL spectra within the same wavelength region from 520 to $530 \mathrm{~nm}$ (i.e., green wavelength region). This result

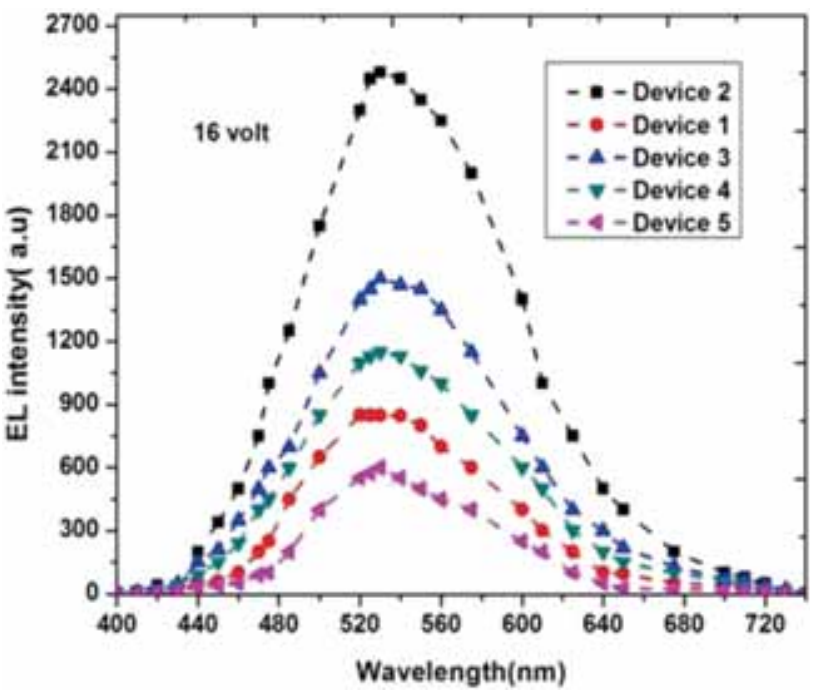

Figure 4. Electroluminescence spectrum of all the OLED devices at $16 \mathrm{~V}$.

shows that the thickness variation buffer layer does not cause the change in the light emission spectrum.

Table 1 shows the summary of the result of five OLED devices along with their standard values (which is a measure of the spread of their efficiency in a set of data at different voltages from their mean values).

From this table, it is seen that the turn-on voltages are 11.2, $6.3,7.2,8.2$ and 9.3 for the devices with a buffer layer in 0,4 , 8,12 and $16 \mathrm{~nm}$, respectively. Thus, the turn-on voltage of 4,8 and $12 \mathrm{~nm}$ is to be lower than that of the device without a buffer layer. However, there is a tendency that the turn-on voltages are increased with the increase in anode layer thicknesses. This is due to the relatively weak modulation of the internal electric field and hence, the larger voltage is dropped across the buffer layer [42]. Also, when the thickness is increased to $16 \mathrm{~nm}$ of the buffer layer, both the value of luminance and luminance efficiency are found to be decreased due to more reduction of a tunnelling mechanism.

The contact behaviour of organic hole-transport layer and FTO electrode also play a significant role in achieving an improved performance of OLED device. In an OLED device, the ohmic contact is possible between organic semiconductor and electrode surface, only if the work function of the metal electrode is close to the HOMO or the LUMO level of an organic semiconductor, which in turn leads to achieve an enhanced charge injection. In this study, performance of $\mathrm{FTO}+\mathrm{MoO}_{3}$ is more ohmic compared with single FTO electrode as shown in figure $1 \mathrm{~b}$. This is the reason of better device efficiency of bilayer anode than that of single FTO electrode within the charge tunnelling region (up to the critical thickness) of hole-injection layer. Also, the modification of the electrode/organic interface by the insertion of a thin layer of $\mathrm{MoO}_{3}$ prevent the direct contact of the metal electrode with the organic TPD layer and thus, preventing the unfavourable chemical reactions between organic and the electrode surface. 
Table 1. Luminance and efficiency characteristics for the devices with different buffer layer thicknesses at the current density of $20 \mathrm{~mA} \mathrm{~cm}^{-2}$.

\begin{tabular}{lccccc}
\hline $\begin{array}{l}\text { Buffer layer } \\
\text { thickness }(\mathrm{nm})\end{array}$ & $\begin{array}{l}\text { Luminance } \\
\left(\mathrm{cd} \mathrm{m}^{-2}\right)\end{array}$ & $\begin{array}{l}\text { Turn-on } \\
\text { voltage }(\mathrm{V})\end{array}$ & $\begin{array}{l}\text { Luminous } \\
\text { efficiency } \\
\left(\mathrm{cd} \mathrm{A}^{-1}\right)\end{array}$ & $\begin{array}{l}\text { Sheet resistance } \\
\text { of bilayer anode }\end{array}$ & $\begin{array}{l}\text { Standard deviation } \\
\text { value of current } \\
\text { efficiency }\end{array}$ \\
\hline 0 & 762 & 11.2 & 3.81 & 21.23 & 0.830 \\
4 & 960 & 6.3 & 4.80 & 19.52 & 1.050 \\
8 & 1030 & 7.2 & 5.15 & 17.32 & 1.150 \\
12 & 1210 & 8.2 & 6.01 & 15.21 & 1.350 \\
16 & 560 & 9.3 & 2.80 & 13.41 & 0.694 \\
\hline
\end{tabular}

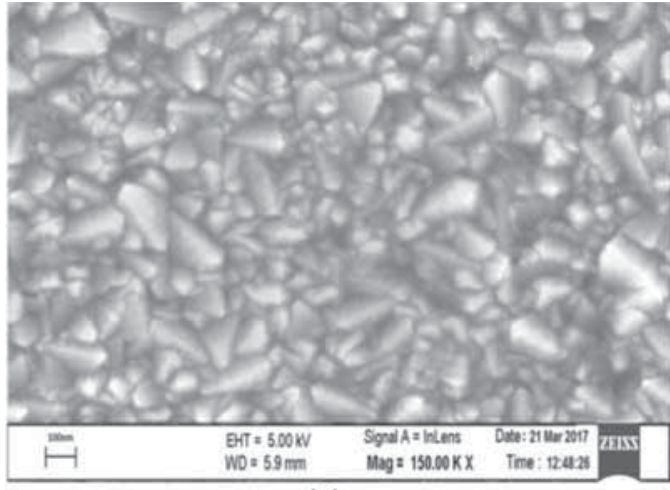

(a)

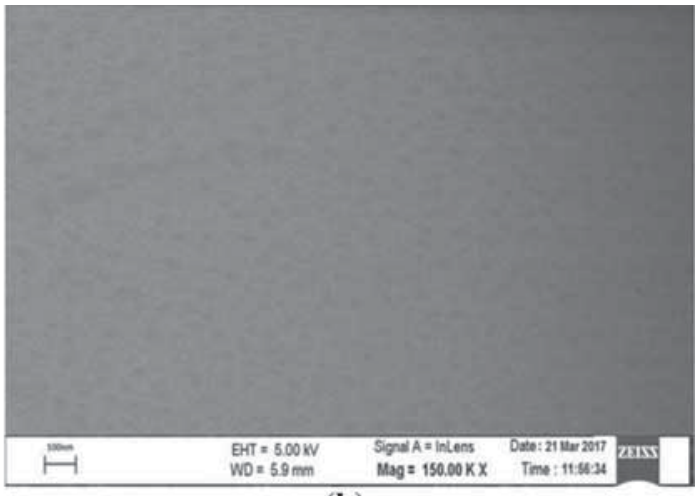

(b)

Figure 5. FE-SEM images of (a) bare FTO surface and (b) $\mathrm{FTO}+\mathrm{MoO}_{3}$ surface.

Along with these, after the insertion of the $\mathrm{MoO}_{3}$ layer over FTO electrode, the surface becomes smoother which is necessary for the achievement of a good OLED device and also for providing a better contact with organic TPD layer. The surface morphology of both the single and double layers of FTO film are shown in figure 5a and b, respectively.

From the above figures, it is clear that the presence of oxide buffer layer smoothens the anode surface by uniformly distributing the whole rough surface region with minimizing surface traps and spikes. This leads to a more homogeneous adhesion of the hole-transporting layer over the entire anode surface. This is an important reason for reducing the contact resistance and hence, the bulk resistance of the device. Because of which we get the better device efficiency when bias is applied to the tunnelling region.

\section{Conclusion}

In summary, the above-presented results demonstrate the fabrication of an organic electro-luminance device on bilayer anode, in which the maximum efficiency is $6.15 \mathrm{~cd} \mathrm{~A}^{-1}$ at the optimized thickness of $12 \mathrm{~nm} \mathrm{MoO}$ interlayer. This result is found to be better than single layer anode under the same condition of operation. Here, we conclude that excessively thick buffer layer leads to high turn-on voltage along with decrease in the current efficiency of the OLED devices due to the high blocking of positive charge carrier injection. In this case, the optimum thickness of $12 \mathrm{~nm} \mathrm{MoO}$ buffer layer has resulted in 1.61 times enhancement in current efficiency compared to the single layer FTO device.

\section{References}

[1] Parker I D 1994 J. Appl. Phys. 751656

[2] Lee H M, Choi K H, Hwang D H, Do L M, Zyung T, Lee J W et al 1998 Appl. Phys. Lett. 722382

[3] Zhou L, Zhuang J Y, Tongay S, Su W M and Cui Z 2013 J. Appl. Phys. 114074506

[4] Mu H, Li W, Jones R, Steckl A and Klotzkin D $2007 \mathrm{~J}$. Lumin. 126225

[5] Meng H, Dai Y, Ye Y, Luo J X, Shi Z J, Dai L et al 2012 J. Phys. D: Appl. Phys. 45245103

[6] Daeil Kim 2013 Trans. Electr. Electron. Mater. 14242

[7] Chien-Jung Huang, Kan-Lin Chen, Po-Wen Sze, Wen-Ray Chen, Teen-Hang Meen and Shi-Lun Wu 2013 Inter. J. Photoenergy 4437304

[8] Hu W P, Manabe K, Furukawa T and Matsumuda M 2002 Appl. Phys. Lett. 802640 
[9] Kabra D, Lu L P, Song M H, Snaith H J and Friend R H 2010 Adv. Mater. 223194

[10] Vasilopoulou M, Palilis L C, Georgiadou D G, Kennou S, Kostis I, Davazoglou D et al P 2012 Appl. Phys. Lett. 100 013311

[11] Hong I, Lee M W, Koo Y M, Jeong H, Kim T S and Song O K 2005 Appl. Phys. Lett. 87063502

[12] Kim T Y, Suh M, Kwon S J, Lee T H, Kim J E, Lee Y J et al 2009 Macromol. Rapid Commun. 301477

[13] Mori T, Fujikawa H, Tokito S and Taga Y 1998 Appl. Phys. Lett. 732763

[14] Zhang S T, Zhou Y C, Zhou J M, Zhan Y Q and Wang Z J 2006 Appl. Phys. Lett. 89043502

[15] Bruner E L, Koch N, Span A R, Bernasek S L, Kahn A and Schwartz J 2002 J. Am. Chem. Soc. 13124

[16] Zhou X L, Sun J X, Peng H J, Mang Z G and Wong M 2005 Appl. Phys. Lett. 1587

[17] Zhang S T, Ding X M, Zhao J M, Shi H Z, He J, Xiong Z H et al 2004 Appl. Phys. Lett. 84425

[18] Lu H T and Yokoyama M 2003 Solid State Electron. 47 1409

[19] Delgertsetseg Byambasuren, Sarangerel Rangerel, Khayankhyarvaa, Javkhlantugs Namsrai, Sakomura Masaru, Ueda Kazuyoshi et al 2014 Rev. Téc. Ing. Univ. 3735

[20] Vasilopoulou M, Papadimitropoulos G, Palilis L C, Georgiadou D G, Argitis P, Kennou S et al 2012 Org. Electron. 13796

[21] Jingze Li, Masayuki Yahiro, Kenji Ishida, Hirofumi Yamada and Kazumi Matsushige 2005 Synth. Met. 151 141

[22] Eliot F Gomez and Andrew J Steckl 2015 ACS Photonics 2 439

[23] Akanksha Uniyal and Poornima Mittal 2016 J. Graphic Era Univ. 432

[24] Yu-Long Wang, Jia-Ju Xu, Yi-Wei Lin, Qian Chen and HaiQuan Shan 2015 AIP Adv. 5107205
[25] Xu Y, Yu H, Wang C et al 2017 Nanoscale Res. Lett. 12 254

[26] Meiling Shan, Haipeng Jiang, Yu Guan, Dongsu Sun, Yu Wang, Jie Hua et al 2017 RSC Adv. 713584

[27] Mu Xue, Wu Xiao-Ming, Hua Yu-Lin, Jiao Zhi-Qiang, Shen Li-Ying and Zheng Jia-Jin 2013 Chin. Phys. B 22027805

[28] Vineeth Michael 2012 PhD Thesis 1 P11287888

[29] Schlatmann A R, Floet D W, Hilberer A, Garten F, Smulders P J M and Klapwijk T M 1996 Appl. Phys. Lett. 691764

[30] Annica Andersson, Nicklas Johansson, Per Broms, NuYu Donald Lupo and William R Salaneck 1998 Adv. Mater. 11859

[31] Ali Kemal Havare, Mustafa Can, Serafettin Demic, Salih Okur, Mahmut Kus, Hasan Aydın et al 2011 Synth. Met. 1612397

[32] Adriano R V, Benvenho Jose P M, Serbena Rudolf Lessmann and Ivo A Hummelgen 2005 Brazilian J. Phys. 351069

[33] Ikeda H, Sakata J, Hayakawa M, Aoyama T, Kawakami T, Kamata K et al 2006 SID Digest 185923

[34] You H, Dai Y, Zhang Z and Ma D 2007 J. Appl. Phys. 101 026105

[35] Mohammad Hossein Habibi and Nasrin Talebian 2005 Acta Chim. Slov. 5253

[36] Andrew M Smith and Shuming Nie 2010 Acc. Chem. Res. 43 190

[37] da Silva M M, Vaz A R, Moshkalev S A and Swart J W 2007 ECS Trans. 9235

[38] Deng Z B, Ding X M, Liao L S, Hou X Y and Lee S T 2000 Display 21323

[39] Deng Z B, Ding X M and Lee S T 1999 Appl. Phys. Lett. 74 2227

[40] Kim J S, Ho P K H, Greenham N C and Friend R H $2002 J$. Appl. Phys. 881073

[41] Bulovic V, Khalfin V B, Gu G, Burrouws P E, Garbuzov D Z and Forrest S R 1998 Phys. Rev. B Condens. Matter 583730

[42] Kim H H, Westerwick E H, Kim Y O, Morris M D, Cerullo M, Miller T M et al 1994 J. Lightwave Technol. 122107 